\title{
Dysfunctional Long-Range Coordination of Neural Activity during Gestalt Perception in Schizophrenia
}

\author{
Peter J. Uhlhaas, ${ }^{1,2}$ David E. J. Linden, ${ }^{1,2,3}$ Wolf Singer, ${ }^{1,4}$ Corinna Haenschel, ${ }^{1,2}$ Michael Lindner, ${ }^{2}$ Konrad Maurer, ${ }^{2}$ and \\ Eugenio Rodriguez ${ }^{1,4}$ \\ ${ }^{1}$ Department of Neurophysiology, Max Planck Institute for Brain Research, Frankfurt am Main, 60528, Germany, ${ }^{2}$ Laboratory for Neurophysiology and \\ Neuroimaging, Department of Psychiatry, Johann Wolfgang Goethe University, Frankfurt am Main, 60528, Germany, ${ }^{3}$ School of Psychology, University of \\ Wales, Bangor LL57 2AS, United Kingdom, and ${ }^{4}$ Frankfurt Institute for Advanced Studies, Johann Wolfgang Goethe University, Frankfurt am Main, 60438, \\ Germany
}

Recent theoretical and empirical research on schizophrenia converges on the notion that core aspects of the pathophysiology of the disorder may arise from a dysfunction in the coordination of distributed neural activity. Synchronization of neural responses in the $\beta$-band $(15-30 \mathrm{~Hz})$ and $\gamma$-band range $(30-80 \mathrm{~Hz})$ has been implicated as a possible neural substrate for dysfunctional coordination in schizophrenia. To test this hypothesis, we examined the electroencephalography (EEG) activity in 19 patients with a Diagnostic and Statistical Manual of Mental Disorder, edition IV criteria, diagnosis of schizophrenia and 19 healthy control subjects during a Gestalt perception task. EEG data were analyzed for phase synchrony and induced spectral power as an index of neural synchronization. Schizophrenia patients were impaired significantly in the detection of images that required the grouping of stimulus elements into coherent object representations. This deficit was accompanied by longer reaction times in schizophrenia patients. Deficits in Gestalt perception in schizophrenia patients were associated with reduced phase synchrony in the $\beta$-band $(20-30 \mathrm{~Hz})$, whereas induced spectral power in the $\gamma$-band $(40-70 \mathrm{~Hz})$ was mainly intact. Our findings suggest that schizophrenia patients are impaired in the long-range synchronization of neural responses, which may reflect a core deficit in the coordination of neural activity and underlie the specific cognitive dysfunctions associated with the disorder.

Key words: schizophrenia; long-range coordination; synchronization; Gestalt perception; electroencephalography; $\beta$-oscillations

\section{Introduction}

Current theories of schizophrenia (Friston, 1998; Andreasen, 1999; Phillips and Silverstein, 2003) have posited that core aspects of the pathophysiology of the disorder arise from a dysfunction in the coordination of distributed neural activity. Synchronous oscillatory activity of neural responses is a possible candidate mechanism for the coordination of neural activity between and within functionally specialized brain regions (Singer, 1999; Varela et al., 2001). There is evidence to suggest that synchronous oscillatory activity is related to feature binding in perception (Rodriguez et al., 1999), working memory (TallonBaudry et al., 1998), and attention (Fries et al., 2001b) and to the preparation and execution of motor acts (Ohara et al., 2001; Schoffelen et al., 2005).

Oscillations in the $\gamma$-frequency $(30-80 \mathrm{~Hz})$ and $\beta$-frequency bands $(15-30 \mathrm{~Hz})$ seem to be of particular relevance for the coordination of neural activity, most likely because these high-

\footnotetext{
Received March 10, 2006; revised June 29, 2006; accepted June 30, 2006.

This work was supported by the Max Planck Society (P.J.U.) and the Hertie Foundation through the Frankfurt Institute of Advanced Studies (E.R.).

Correspondence should be addressed to Dr. Peter J. Uhlhaas, Department of Neurophysiology, Max Planck Institute for Brain Research, Deutschordenstrasse 46, Frankfurt am Main, 60590, Germany. E-mail: uhlhaas@mpih-frankfurt.mpg.de.

D0I:10.1523/JNEUROSCI.2002-06.2006

Copyright $\odot 2006$ Society for Neuroscience $\quad$ 0270-6474/06/268168-08\$15.00/0
}

frequency oscillations are particularly effective in supporting precise synchronization of neuronal discharges (Fries et al., 2001a).

Recent studies with electroencephalography (EEG) have provided evidence that patients with schizophrenia may be characterized by abnormalities in neural synchronization. The research so far has focused on the analysis of spectral power (Gallinat et al., 2004; Spencer et al., 2004), which measures only the activity of the spatial summation of cortical responses in the range of $1 \mathrm{~cm}$, thus assessing essentially intra-areal coordination (Varela et al., 2001). In contrast, long-range coordination involves neural assemblies that are farther apart in the brain $(>2 \mathrm{~cm}$ ) (Varela et al., 2001). Long-range coordination in EEG data can be examined by testing the stability of phase differences between electrodes (Lachaux et al., 1999). Recent evidence (Kopell et al., 2000) suggests that oscillations in the $\beta$ - and lower $\gamma$-frequency range may be particularly suited for long-range coordination, because synchronization at lower frequencies tolerates longer conduction delays than $\gamma$-rhythms.

There is preliminary evidence that patients with schizophrenia may be characterized by dysfunctional long-range coordination (Spencer et al., 2003; Slewa-Younan et al., 2004; Symond et al., 2005). However, the research has examined phase synchrony only in a narrow frequency range in the $\gamma$-band $(37-44 \mathrm{~Hz})$ and has relied on experimental tasks that did not produce a clear behavioral impairment in patients with schizophrenia. Thus the 
Table 1. Demographic and clinical characteristics of schizophrenia patients and controls

\begin{tabular}{|c|c|c|c|c|c|}
\hline \multirow[b]{2}{*}{ Variable } & \multicolumn{2}{|c|}{ Schizophrenia $(n=19)$} & \multicolumn{2}{|c|}{ Controls $(n=19)$} & \multirow[b]{2}{*}{ Significance level } \\
\hline & M & SD & M & SD & \\
\hline Age (years) & 35.2 & 9.7 & 30.1 & 9.5 & $\begin{aligned} t_{(36)} & =1.61 \\
p & =0.11\end{aligned}$ \\
\hline Sex (male/female) & $12 / 7$ & & $14 / 5$ & & $\begin{aligned} \chi^{2}(1) & =0.133 \\
p & =0.49\end{aligned}$ \\
\hline Education (years) & 15.1 & 3.3 & 16.2 & 2.6 & $\begin{array}{c}t_{(36)}=-1.12 \\
p=0.26\end{array}$ \\
\hline Verbal IQ & 107.4 & 15.0 & 112.1 & 14.4 & $\begin{array}{c}t_{(36)}=-0.90 \\
p=0.37\end{array}$ \\
\hline Laterality index & 66.4 & 34.6 & 54.5 & 65.4 & $\begin{aligned} t_{(36)} & =0.64 \\
p & =0.53\end{aligned}$ \\
\hline Duration of illness & 7.9 & 7.0 & & & \\
\hline \multicolumn{6}{|c|}{ Positive and negative syndrome scale scores } \\
\hline Excitement & 11.3 & 2.8 & & & \\
\hline Negative & 13.6 & 4.9 & & & \\
\hline Cognitive & 8.5 & 2.6 & & & \\
\hline Positive & 8.1 & 3.4 & & & \\
\hline Depression & 11.4 & 3.0 & & & \\
\hline
\end{tabular}

a)

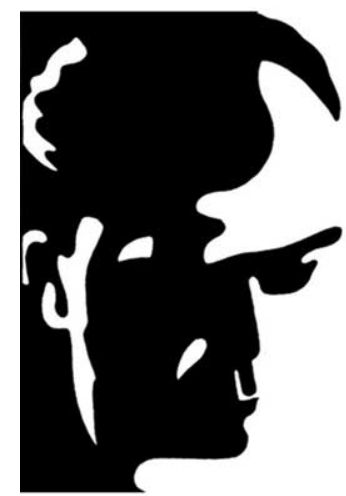

b)

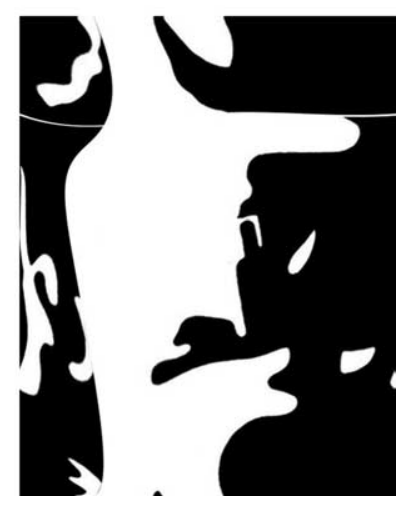

Figure 1. Shown are an upright Mooney face $(\boldsymbol{a})$ and an inverted version of the same image $(\boldsymbol{b})$.

extent to which patients with schizophrenia are characterized by dysfunctional synchronization of neural responses and the involvement of abnormal synchronization in cognitive deficits are still unresolved questions.

In previous studies we have demonstrated that the perception of Mooney faces (see Fig. 1) (Mooney and Ferguson, 1951) correlates with increased phase synchrony in healthy individuals (Rodriguez et al., 1999) and that patients with schizophrenia are impaired in the grouping of stimulus elements into coherent object representations (Uhlhaas and Silverstein, 2005; Uhlhaas et al., 2005). On the basis of these results we hypothesized that schizophrenia patients would show deficits in the perception of upright Mooney faces because these depend on Gestalt perception. We expected that the behavioral dysfunctions in Gestalt perception in patients with schizophrenia would be associated with reductions in phase synchrony because it reflects precise temporal coordination of distributed neural activity.

\section{Materials and Methods}

Participants. Nineteen subjects with schizophrenia were recruited from inpatient and outpatient units from the Frankfurt University Psychiatry Department. Nineteen healthy controls were recruited from the local community. Written informed consent was obtained from all participants after a description of the study procedures was given. Demographic information for patients and controls is given in Table 1. Patients with schizophrenia and control subjects were of similar age and education. No differences between groups were found for premorbid verbal IQ and handedness. Via the Diagnostic and Statistical Manual of Mental Disorders, edition IV criteria (DSMIV), the diagnosis of schizophrenia was established with the German version of the Structured Clinical Interview for DSM-IV (Sass and Wittchen, 2003), by thorough chart review, and in consultation with the treating psychiatrists. Current psychopathology was assessed with the positive and negative syndrome scale (PANSS) (Kay et al., 1986), and symptoms were grouped into five factors according to the model of Lindenmayer et al. (1995).

Patients and controls were excluded if they had any neurological or ophthalmologic disorders that might have affected performance or if they met criteria for alcohol or substance dependence within the last month. In addition, the controls were screened with the Symptom Checklist-90-Revised (SCL-90-R) (Franke, 1995) for psychiatric disorders and possible psychotic episodes. All patients were on medication at the time of testing, with 18 of 19 receiving atypical antipsychotic medication (olanzapine, 5; amisulpride, 4; clozapine, 4; risperidone, 3; ziprasidone, 1 ; aripiprazole, 1 ). The medication dosage measured in chlorpromazine equivalents (Woods, 2003) was $714 \mathrm{mg} / \mathrm{d}$. Visual acuity was examined with a standard visual acuity examination (Snellen Chart), which involved examining acuity monocularly in each eye and then binocularly.

Stimuli and task. Mooney and Ferguson (1951) developed a visual closure task consisting of degraded pictures (Fig. 1) in which all shades of gray are removed, thereby leaving the shadows rendered in black and the highlights in white. The test contains 51 degraded black and white images of men, women, and children of various ages. Perception of Mooney faces involves the grouping of the fragmentary parts into coherent images, based on the Gestalt principle of closure (Mooney and Ferguson, 1951).

For the experimental condition 44 Mooney faces were selected in their original orientation and once after vertical mirroring (face condition). These stimuli also were presented upside-down (no-face condition). In addition, inverted Mooney faces were scrambled by selecting various stimulus features to decrease the likelihood of perceiving a face in inverted stimuli. All stimuli subtended a visual angle of $\sim 7 \times 10^{\circ}$.

Stimuli were presented on a 19 inch computer screen. A fixation cross was presented in the center of the screen between trials. After a training block of eight trials (four stimuli not included in the experimental block) the participants received four blocks of 88 experimental trials. Each block consisted of 44 upright and 44 inverted stimuli. The stimuli were presented in a controlled random order, ensuring that a given Mooney face (upright, inverted vertically mirrored, or original orientation) appeared only once in each block. Each stimulus was presented once in each combination of orientation and facing direction over the first two blocks. The next two blocks were the exact repetition of the first two blocks. Stimuli were presented for $200 \mathrm{~ms}$ with an interstimulus interval of 3000-4500 ms.

The participants' task was to report whether a face had been perceived, regardless of orientation, by pressing one of two response buttons mounted on a response pad. Participants were instructed to respond as quickly as possible but were told to press the "face" button only if they perceived a whole face.

Electrophysiological recording and analysis. Electroencephalographic activity was recorded from 63 scalp sites, using the BrainAmp amplifier (Brain Products, Munich, Germany) and Braincap electrode cap (Falk Minow Services, Munich, Germany). The electrodes were placed according to the extended 10-20 System. All channels were referenced during 
recording to an electrode (fronto-central position, $\mathrm{FCz}$ ) with a forehead ground and impedance of $<5 \mathrm{k} \Omega$. An additional electrode was placed on the infraorbital ridge of the right eye to record the vertical electro-oculogram (EOG). The EEG and EOG were digitized with a sampling rate of $500 \mathrm{~Hz}$. The initial bandpass recording filter was set at $0.01-100 \mathrm{~Hz}$.

For the analysis the EEG data were referenced to electrodes TP9-TP10. This reference was chosen because it was unlikely to be involved in widespread synchronous activity. Principal component analysis (PCA) (Ille et al., 2002) was used to identify eyeblink artifacts by their distinct topography and to remove their contribution from a subject's data. Then the digitized signals were analyzed by means of a windowed Fourier transform (window length, 192 ms; step, 20 ms; window overlap, $90 \%)$. Signal windows were zero-padded to 512 points to obtain an interpolated frequency resolution of $\sim 1 \mathrm{~Hz} /$ frequency bin.

For every time window and frequency bin the amplitude and phase were computed as follows: if we let $C(f, t)_{\mathrm{r}}$ and $C(f, t)_{\mathrm{i}}$ be the real and imaginary Fourier coefficients obtained for frequency $f$ and time window $t$, then the amplitude of the signal at time $t$ and frequency $f$ is given by $\operatorname{Amp}(f, t)=\operatorname{sqrt}\left[C(f, t)_{\mathrm{r}}{ }^{2}+C(f, t)_{\mathrm{i}}{ }^{2}\right]$.

This amplitude is thus the length of the vector specified by the real and imaginary Fourier coefficient computed by Pythagoras' theorem. It corresponds to the magnitude of the observed oscillation at a given time and frequency point.

The phase of the signal at the same time and frequency is as follows:

$$
\phi(f, t)=\operatorname{arctg}\left[C(f, t)_{\mathrm{i}} / C(f, t)_{\mathrm{r}}\right] .
$$

Thus $\phi$ is the angle of the vector specified by the real and imaginary Fourier coefficient. For a given time and frequency point it corresponds to the position inside the oscillation cycle (peak, valley, or rising or falling slope).

These amplitude and phase values were evaluated in the $15-80 \mathrm{~Hz}$ frequency range and in the -800 to $+1000 \mathrm{~ms}$ period relative to stimulus onset. The phase information was used to compute a time-varying phaselocking value (PLV) as an index of neural synchrony as reported previously (Lachaux et al., 1999). In brief, the method involves computing the phase difference inside a time window between all electrode pairs and then assessing the stability of such phase differences through all of the trials in the following manner.

We let $\phi_{\mathrm{i}}$ and $\phi_{\mathrm{j}}$ be unitary vectors representing the phase of signals in electrodes $i$ and $j$ during time window $t$ and frequency bin $f$. The phase difference between such electrodes is given by $\phi_{\mathrm{ij}}=\phi_{\mathrm{i}} \operatorname{conj}\left(\phi_{\mathrm{j}}\right)$, and the phase-locking value is $\mathrm{PLVij}=\mathrm{abs}\left(1 / N \times \sum \phi_{\mathrm{ij}}\right)$, with the sum operating through all of the trials and $N$ being the number of trials.

Statistical analysis. Because we were interested in long-range coordination of neural activity, we included all electrodes in the calculation to produce a global index of synchronization across a large frequency range. Furthermore, only trials in which subjects responded correctly were considered for analysis. Behavioral and EEG data were analyzed with twotailed $t$ tests. The alpha level was set at 0.05 for all tests.

Time frequency charts of both phase synchrony and spectral power were normalized to a baseline of $800 \mathrm{~ms}$ preceding the stimulus onset. The normalization involves subtracting the baseline average and dividing by the baseline SD on a frequency by frequency basis in the following manner: if we let $S$ be a signal, $\mu$ the average of the signal during the baseline period, and $\sigma$ the SD of the same baseline period, then the normalized signal is given by $S_{\mathrm{N}}=(S-\mu) / \sigma$.

For the topographical analysis of phase synchrony we controlled for the statistical effects of multiple comparisons by choosing a very conservative significance threshold $(p<0.0005)$. This threshold was set on the basis of the distribution of synchrony values during the baseline. The threshold was chosen such that the number of cases larger than the threshold divided by the total number of cases was equal to $p=0.00005$. By choosing this significance level, one line per analysis window could be explained by chance, given the fact that there were 62 electrodes with 1891 possible combinations $[(62 \times 61) / 2=1891]$.

\section{Results}

\section{Behavioral results}

We analyzed the percentage of correct and incorrect responses as well as reaction times (RTs) for the face and no-face conditions (Table 2). Responses were classified as correct when subjects recognized upright faces as "face" and inverted faces as "no face." Schizophrenia patients perceived significantly fewer faces in the face condition than controls $\left(t_{(36)}=3.19 ; p=0.03\right)$. Moreover, schizophrenia patients were significantly slower in RTs than normal controls in the face condition $\left(t_{(36)}=2.34 ; p=0.03\right)$. In the no-face condition no differences between patients with schizophrenia and controls were found for the percentage of correct responses $\left(t_{(36)}=0.78 ; p=0.76\right)$ or $\mathrm{RTs}\left(t_{(36)}=0.76 ; p=0.45\right)$.

We furthermore examined the relationship among behavioral data, PANSS ratings, medication, and demographic variables. A significant negative correlation between the number of correct responses in the no-face condition and symptoms of the factor "positive" of the PANSS was found $(-0.51 ; p=0.03)$. In addition, the correlation between RTs in the face condition and elevated symptom scores for the factor "negative" approached statistical significance $(0.46 ; p=0.05)$. No other significant correlations among RTs, percentage of correct responses, and factors of the PANSS were obtained. In addition, there were no significant correlations among percentage of correct responses, RTs, and demographic variables (age, verbal IQ, illness duration, age of onset). Finally, antipsychotic medication dosage in chlorpromazine equivalents was not correlated with RTs (face condition, -0.03 and $p=0.90$; no-face condition, -0.12 and $p=0.61$ ) nor with percentage of correct responses (face condition, 0.13 and $p=0.59$; no-face condition, 0.26 and $p=0.29$ ) in patients with schizophrenia.

\section{EEG data}

For the schizophrenia patients the mean number of trials per subject that were entered into the analysis was $92.3(\mathrm{SD}=28.4)$ in the face and $113.8(\mathrm{SD}=28.5)$ in the no-face conditions. For controls there were 101.6 ( $\mathrm{SD}=37.2)$ trials in the face and 120.9 $(S D=13.2)$ trials in the no-face conditions. The difference for the number of trials between groups in the face condition was statistically significant $\left(t_{(36)}=3.92 ; p=0.0001\right)$, but not in the no-face condition $\left(t_{(36)}=1.14 ; p=0.26\right)$.

\section{Time frequency analysis}

We analyzed oscillatory activity in the $\beta$-band $(15-30 \mathrm{~Hz})$ and in the $\gamma$-band $(30-80 \mathrm{~Hz})$ within a $150-400 \mathrm{~ms}$ interval that has 
a)
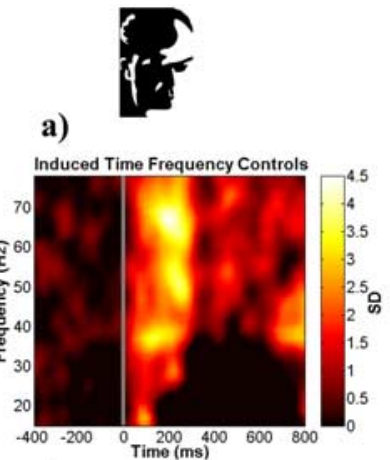

c)

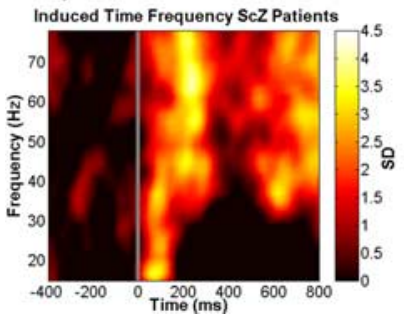

b)
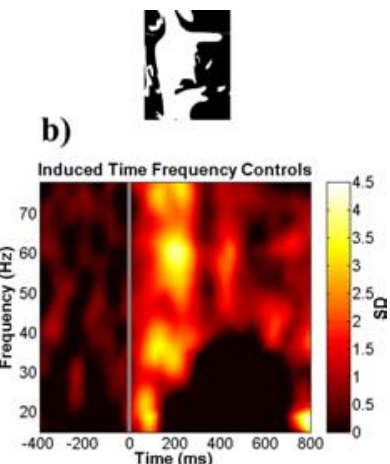

d)

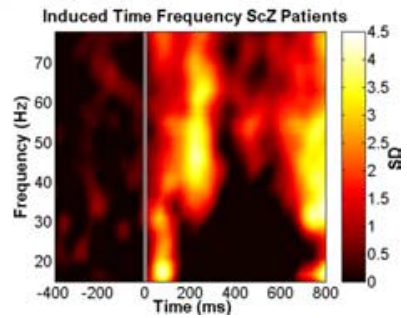

Figure 2. Group average of induced spectral power analysis for all electrodes and correct trials. $\boldsymbol{a}$, Face condition for controls. $\boldsymbol{b}$, No-face condition for controls. $\boldsymbol{c}$, Face condition for patients with schizophrenia. $\boldsymbol{d}$, No-face condition for patients with schizophrenia

been related to cognitive processes involved in the construction of coherent object representations (Rodriguez et al., 1999; Tallon-Baudry and Bertrand, 1999).

\section{$\gamma$-Band}

For controls the $\gamma$-band activity in the face condition peaked between 200 and $300 \mathrm{~ms}$ after stimulus onset in the $40-70 \mathrm{~Hz}$ frequency range, with a maximum amplitude between 60 and 70 $\mathrm{Hz}$ (Fig. 2a). $\gamma$-Band activity in the no-face condition was similar to induced spectral power in the face condition (Fig. 2b) (frequency range, $40-70 \mathrm{~Hz}$; time interval, $200-300 \mathrm{~ms}$; $t_{(36)}=0.55$; $p=0.58)$.

Patients with schizophrenia showed a similar pattern of induced spectral power during the face (Fig. $2 c$ ) and no-face conditions (Fig. $2 d$ ) as compared with controls (face condition: frequency range, $40-70 \mathrm{~Hz}$; time window, $200-300 \mathrm{~ms} ; t_{(36)}=0.03$; $p=0.98$ ) (no-face condition: frequency range, $40-70 \mathrm{~Hz}$; time interval, $\left.200-300 ; t_{(36)}=-0.51 ; p=0.61\right)$.

\section{$\beta$-Band}

For the $\beta$-frequency band we did not find significant oscillatory activity during the perception period.

\section{Phase synchronization}

The main focus of our work was the analysis of phase synchrony. In contrast to the analysis of spectral power, phase synchrony is a direct measure of synchronization in the EEG that is not confounded by the amplitude of the signal (Lachaux et al., 1999).

\section{$\beta$-Band}

In contrast to the time frequency analysis, phase synchrony for the face condition in controls exhibited two maxima over an average frequency range of $20-30 \mathrm{~Hz}$ (Fig. $3 a$ ). The first peak occurred $\sim 200 \mathrm{~ms}$ after stimulus onset and the second peak at $\sim 400-600 \mathrm{~ms}$. Statistical analysis of the face and no-face conditions yielded a significant increase in phase synchrony in the face condition as compared with the no-face condition (frequency

a)

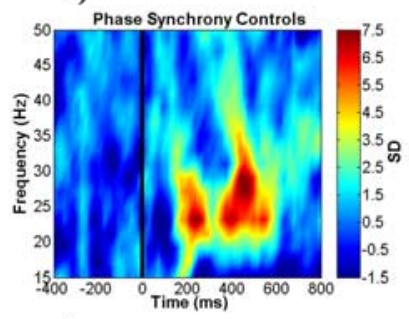

c)

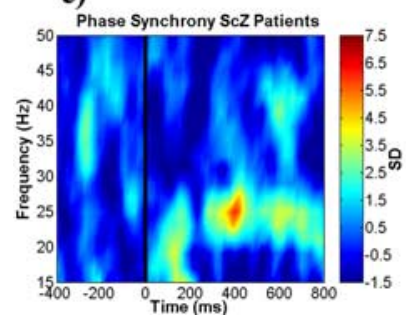

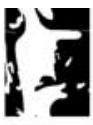

b)

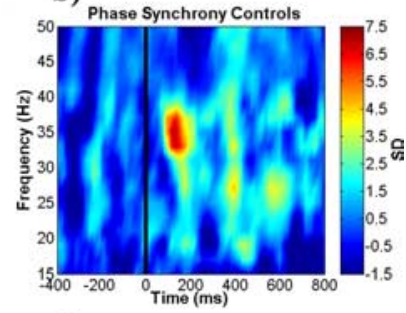

d)

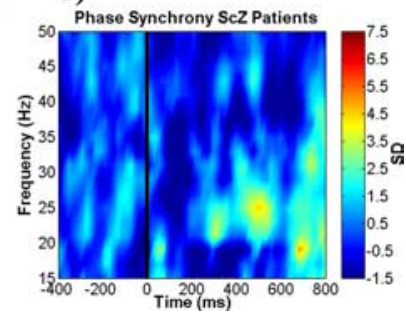

Figure 3. Group average of phase synchrony for all electrodes and correct trials. a, Face condition for controls. $\boldsymbol{b}$, No-face condition for controls. $\boldsymbol{c}$, Face condition for patients with schizophrenia. $\boldsymbol{d}$, No-face condition for patients with schizophrenia.

range, $20-30 \mathrm{~Hz}$; time interval, $200-280 \mathrm{~ms} ; t_{(36)}=2.52 ; p=$ $0.01)$.

In patients with schizophrenia the onset of the first peak in the face condition was delayed and occurred between 350 and $400 \mathrm{~ms}$ in the frequency range of $20-25 \mathrm{~Hz}$ (Fig. 3c). In addition, a second, weaker peak was found at $\sim 600 \mathrm{~ms}$. When compared with controls, the reduction in phase synchrony in the frequency range of $20-30 \mathrm{~Hz}$ was significant (frequency range, $20-30 \mathrm{~Hz}$; time interval, $\left.200-280 \mathrm{~ms} ; t_{(36)}=2.96 ; p=0.005\right)$.

To rule out the possibility that the differences in phase synchrony between controls and patients with schizophrenia were attributable to a circumscribed deficit in face perception, we performed an analysis in which phase synchrony patterns involving electrodes P9/P10 were removed from the analysis. These electrodes commonly are used for the EEG analysis of face perception (Onitsuka et al., 2006). The difference between patients with schizophrenia and controls in the face condition remained statistically significant (frequency range, $20-30 \mathrm{~Hz}$; time interval, $\left.200-280 \mathrm{~ms} ; t_{(36)}=3.47 ; p=0.001\right)$.

$\gamma$-Band

In the face condition the recordings from the control group showed no significant activity in the frequency range in which we observed induced oscillatory activity. There was an early peak of $\gamma$-band activity (time interval, 120-170 ms; frequency range, $31-38 \mathrm{~Hz}$ ) in controls that was, however, not significantly different from activity in the no-face condition (time interval, 120-170 $\mathrm{Hz}$; frequency range, $31-38 \mathrm{~Hz} ; t_{(36)}=-0.80 ; p=0.42$ ).

In patients with schizophrenia we observed a pronounced desynchronization in the $\gamma$-band $(30-55 \mathrm{~Hz})$ in the face condition in the 200-280 ms interval that was not present in the control group (Fig. 3c) (frequency range, 30-55 Hz; time interval, $200-$ $\left.280 \mathrm{~Hz} ; t_{(36)}=2.04 ; p=0.04\right)$. In the no-face condition the patients with schizophrenia also showed a significant reduction in $\gamma$-band activity between 120 and $170 \mathrm{~ms}$ as compared with controls (Fig. 3d) (frequency range, 31-38 Hz; time interval, $\left.120-170 \mathrm{~ms} ; t_{(36)}=2.71 ; p=0.01\right)$. 
We also observed phase synchrony in the $\beta$-band in controls in a later interval between 400 and $600 \mathrm{~ms}$ that previously has been related to the preparation and execution of motor responses (Rodriguez et al., 1999), but no statistically significant differences were observed between groups (face condition: frequency range, 25-30 $\mathrm{Hz}$; time interval, $420-500 \mathrm{~ms} ; t_{(36)}=$ $1.75 ; p=0.08$ ) (no-face condition: frequency range, 25-30 Hz; time interval, $\left.420-500 \mathrm{~ms} ; t_{(36)}=-0.45 ; p=0.65\right)$.

Topographical analysis of phase synchrony

To obtain detailed spatiotemporal information on phase synchrony deficits in the $\beta$-band in patients with schizophrenia during Gestalt perception, we computed the regional distribution of phase synchrony over the scalp for the face condition for a $0-400 \mathrm{~ms}$ interval in the $20-30$ $\mathrm{Hz}$ frequency range (Fig. 4). PLVs between electrodes were computed for every pair of electrodes and for a given time and frequency window to define a synchrony pattern. This synchrony pattern can be displayed as a network of lines connecting the corresponding electrodes to indicate synchronous neural activity between such electrodes during a given time window. Synchrony lines are displayed only if the probability is $p<0.00005$.

In controls we found an increase in phase synchrony after $200 \mathrm{~ms}$ that involved interactions among temporal, frontal, and parieto-occipital electrodes. In contrast, patients with schizophrenia were characterized by an absence of phase synchrony in the 200-300 ms interval. Specifically, patients with schizophrenia showed reduced synchrony patterns between fronto-temporal and parieto-occipital electrodes as compared with controls.

\section{Topographical analysis of induced}

$\boldsymbol{\gamma}$-band power

Controls showed an increase in induced spectral power between 40 and $70 \mathrm{~Hz}$ after $100 \mathrm{~ms}$ that involved mainly bilateral parieto-occipital electrodes as well activity over right frontal electrodes (Fig. 5). Similar activity was observed in the $200-300 \mathrm{~ms}$ interval. Patients with schizophrenia were characterized by a reduction in amplitude as compared with controls over occipitoparietal (PO3, POz, PO4, O1, Oz, O2), left temporal (T7, TP7, TP9), and right frontal electrodes (AF4, F2, F4, F6, FC2). These differences, however, were not statistically significant (occipito-parietal electrodes: 65-75 Hz; $190-270 \mathrm{~ms} ; t_{(36)}=1.51 ; p=0.16$ ) (left temporal electrodes: $53-67$ $\mathrm{Hz} ; 200-255 \mathrm{~ms} ; t_{(36)}=1.71 ; p=0.19$ ) (right frontal electrodes: $\left.49-65 \mathrm{~Hz} ; 200-270 \mathrm{~ms} ; t_{(36)}=1.23 ; p=0.29\right)$.
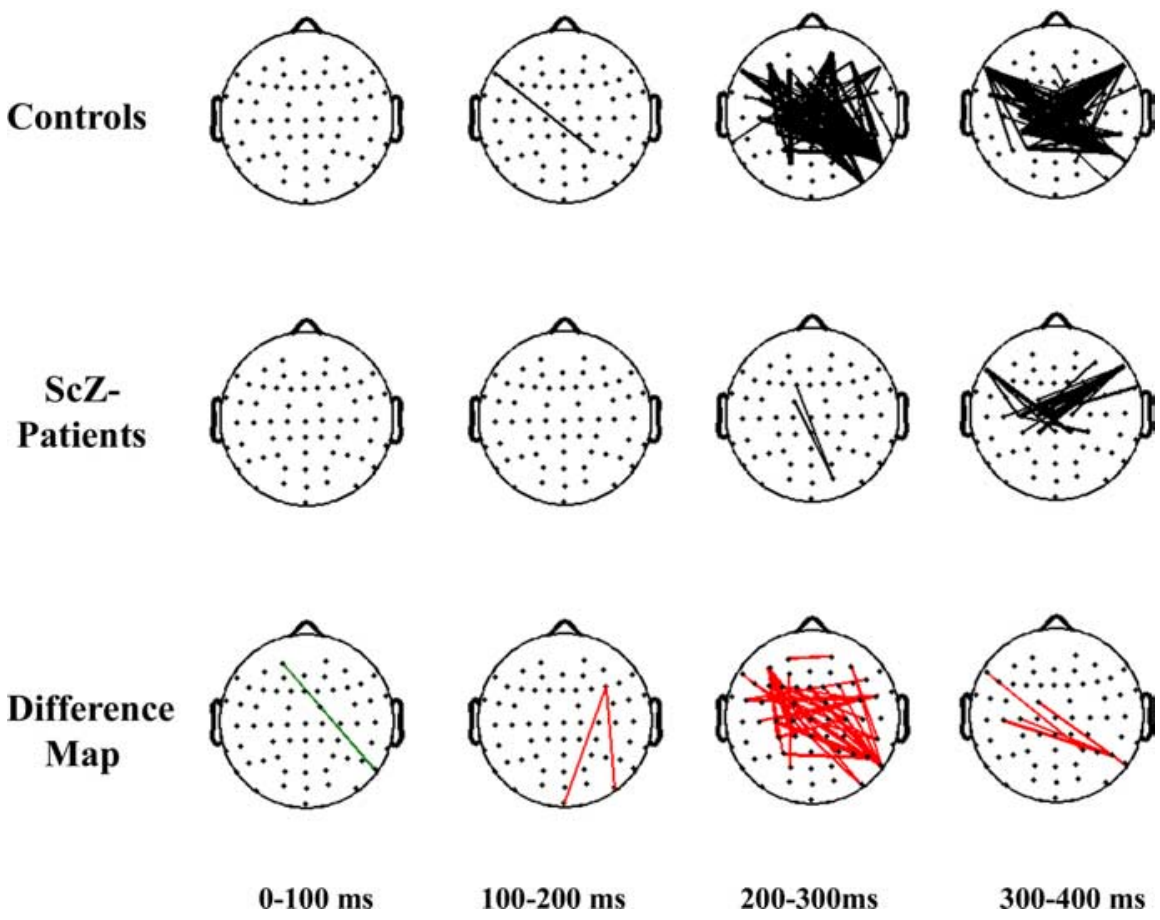

Figure 4. Topography of phase synchrony for face condition. Top row, Controls. Middle row, Patients with schizophrenia. Bottom row, Difference map. Synchrony between electrodes is indicated by lines, which are drawn only if the synchrony value is beyond a two-tailed probability of $p<0.0005$. Differences between groups are displayed in the bottom row. Red lines indicate a decrease in synchrony for patients with schizophrenia compared with controls. Green lines indicate an increase in synchrony for patients with schizophrenia relative to controls.
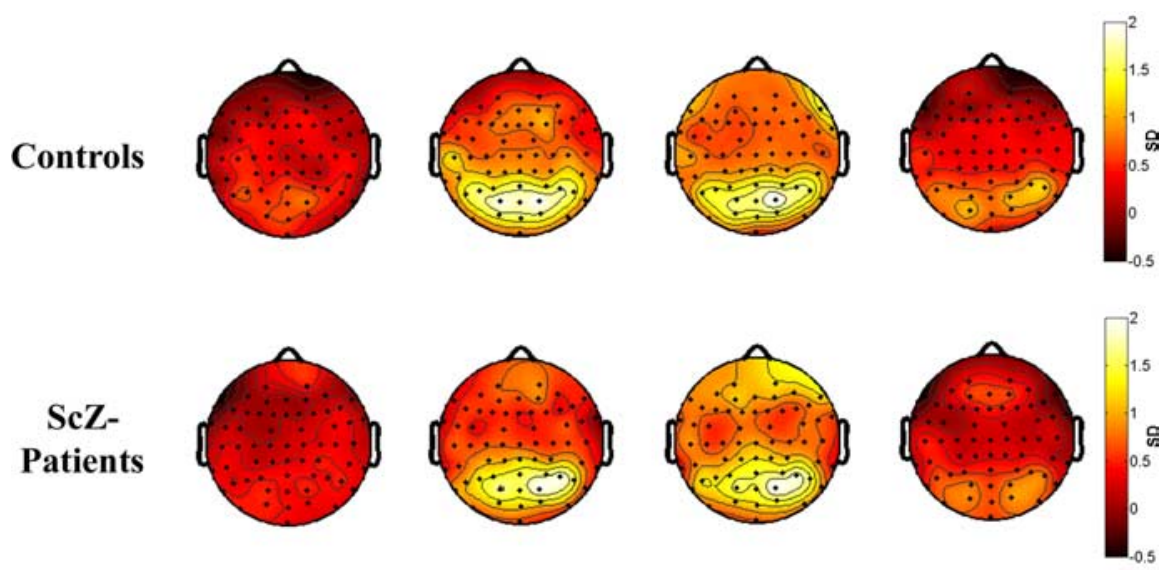

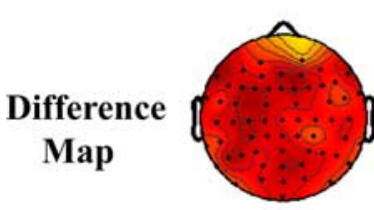

0-100 ms

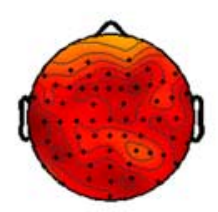

100-200 ms

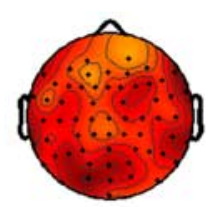

200-300ms

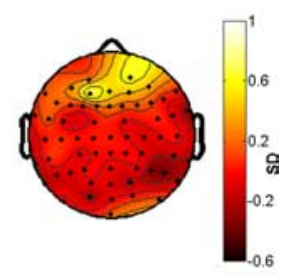

$300-400 \mathrm{~ms}$
Figure 5. Topography of induced spectral power for face condition. Top row, Controls. Middle row, Patients with schizophrenia. Bottom row, Difference map. Amplitude is given in SDs in reference to the baseline.

EEG data, clinical symptoms, medication, and demographic variables

We examined the relationship among EEG parameters, clinical symptoms, medication, and demographic variables. No significant correlations were found for induced spectral power in the 
face and no-face conditions (frequency range, 40-70 Hz; time interval, 200-300 ms) and clinical symptoms as assessed by the PANSS and demographic variables (age, verbal IQ, illness duration, and age of onset). In addition, there was no correlation with antipsychotic medication dosage and induced spectral power (face condition, 0.16 and $p=0.95$; no-face condition, -0.08 and $p=0.75)$. In the next step we computed correlations for phase synchrony values in the face and no-face conditions for time windows in which schizophrenia patients showed significant deficits relative to controls. For the time window in the face condition (frequency range, $20-30 \mathrm{~Hz}$; time interval, 200-300 ms) we obtained a significant correlation between elevated scores on the factor positive and increased phase synchrony $(0.48 ; p=0.04)$. Specifically, increased phase synchrony correlated with the items "delusions" $(0.51 ; p=0.02)$ and "hallucinations" $(0.55 ; p=$ $0.01)$. For the no-face condition there was a significant correlation between elevated scores of the factor negative and reduced phase synchrony $(-0.56 ; p=0.005)$ as well as a statistical trend for a correlation between increased positive symptoms and reduced phase synchrony $(-0.40 ; p=0.07)$. Dysfunctions in phase synchrony in patients with schizophrenia in both experimental conditions were not correlated with duration of illness, age of onset, nor with antipsychotic medication dosages (face condition, 0.13 and $p=0.57$; no-face condition, 0.16 and $p=0.51$ ).

\section{Discussion}

The results of this study support the hypothesis that patients with schizophrenia are characterized by dysfunctions in long-range coordination of neural activity, as indicated by significant reductions in phase synchrony during Gestalt perception. These findings are relevant for current concepts of schizophrenia, which emphasize that core aspects of the pathophysiology of the disorder arise from a deficit in the coordination of distributed brain activity (Friston, 1998; Andreasen, 1999; Phillips and Silverstein, 2003). Moreover, the close correspondence between behavioral deficits in Gestalt perception and reduced neural synchronization, which we observed in our data, supports the role of impaired phase synchrony as an underlying mechanism of dysfunctional cognition in schizophrenia.

\section{Phase synchrony and Gestalt perception}

In agreement with previous empirical data (Rodriguez et al., 1999; Trujillo et al., 2005) we found that phase synchrony distinguished between the processing of face and no-face stimuli. Stimulus processing in the face condition involved long-range interactions in the $\beta$-frequency range $(20-30 \mathrm{~Hz})$ among frontal, temporal, and parietal electrodes, suggesting that the formation of a coherent object representation is related to large-scale neural interactions. Differences in induced spectral power were not clearly present between stimuli in the face and no-face conditions. This suggests that processes involving local synchronization of oscillatory activity are less affected than processes requiring long-range synchronization during Gestalt perception.

The differential roles of phase synchrony and induced spectral power in the coordination of neural activity also are supported by the distinct frequency band in which activity during Gestalt perception was observed. Whereas induced spectral power was found predominantly in the $\gamma$-band range $(40-70 \mathrm{~Hz})$, phase synchrony occurred in a lower-frequency range, mainly between 20 and $30 \mathrm{~Hz}$, which corresponds to the $\beta$-frequency. This finding is consistent with previous research that has found that longrange coordination occurs in the $\beta$ - and lower $\gamma$-band range (Roelfsema et al., 1997; Rodriguez et al., 1999; Kopell et al., 2000;
Bibbig et al., 2002; Brovelli et al., 2004; Gross et al., 2004; TallonBaudry et al., 2004).

\section{Long-range neural coordination and schizophrenia}

The hypothesis that the pathophysiology of schizophrenia involves a fundamental deficit in long-range coordination of neural activity is supported strongly by the differential impairments that we observed in phase synchrony and induced spectral power. Patients with schizophrenia were impaired particularly in phase synchrony in the $\beta$-frequency range during Gestalt perception in time windows that differentiated between face and no-face conditions in controls. In fact, patients with schizophrenia were characterized by tendencies to exhibit desynchronization of neural activity, suggesting a marked dysfunction not only in the initiation but also in the maintenance of synchronous oscillatory activity.

Dysfunctional coordination of neural activity in patients with schizophrenia is highlighted furthermore by the topographical analysis of phase synchrony (Fig. 4). Synchrony patterns in the $\beta$-frequency range in patients with schizophrenia were mainly absent in the 200-300 ms interval during which controls showed the largest number of synchrony patterns, suggesting that patients with schizophrenia failed to coordinate neural activity among temporal, frontal, parietal, and occipital brain regions to produce a fully integrated percept.

In contrast to the phase synchrony activity in the $\beta$-frequency band, patients with schizophrenia showed no major changes in induced spectral power in the $\gamma$-band range. We observed reductions only in induced spectral power over selected frontal, parieto-occipital, and temporal electrodes during the processing of stimuli in the face condition, suggesting that local coordination processes, which are reflected by induced $\gamma$-band power, might be relatively preserved in patients with schizophrenia.

\section{Cognitive dysfunctions and phase synchrony in schizophrenia}

The role of impaired phase synchrony between 20 and $30 \mathrm{~Hz}$ as an underlying mechanism of dysfunctional cognition in schizophrenia is supported by the close correspondence between behavioral deficits in Gestalt perception and the reduction in phase synchronization that we observed in our data. First, patients with schizophrenia demonstrated a selective deficit in Gestalt perception that was present only when stimulus elements had to be grouped into a meaningful percept. The dysfunction in Gestalt perception in the present study was accompanied by a reduction in phase synchrony that was largest in the face condition during time windows that differentiated responses to face and no-face stimuli in controls, suggesting that dysfunctions in phase synchrony have a causal role for the explanations of cognitive deficits in schizophrenia. This dysfunction is unlikely to reflect a specific impairment for faces (Onitsuka et al., 2006), because patients with schizophrenia are characterized by a basic dysfunction in the integration of stimulus elements into coherent object representations that is present for a wide range of stimuli (Uhlhaas and Silverstein, 2005). The reduction in phase synchrony in the $\beta$-frequency range also remained statistically significant after electrodes were removed for which the signals had been linked to face perception.

Contrary to our hypothesis, however, we also observed a significant reduction in phase synchrony during the no-face condition in patients with schizophrenia, which occurred within a narrower frequency band and time window than in the face condition, indicating a generalized impairment in large-scale co- 
ordination of distributed activity. Phenomenological and psychophysical data (Uhlhaas and Silverstein, 2005) have shown that dysfunctions in Gestalt perception in patients with schizophrenia lead to the processing of irrelevant information. In the present study the absence of the early phase synchrony response may be interpreted in terms of a dysfunction in early grouping mechanisms that forces the subjects to seek solutions by the serial analysis of visual information. This hypothesis is supported by the fact that patients with schizophrenia had longer reaction times than controls in the face condition and smaller reaction time differences between the face and no-face conditions.

\section{Phase synchrony and the symptoms of schizophrenia}

The current data also allow for preliminary conclusions on the relationship between deficits in phase synchrony and the symptoms of schizophrenia. Phase synchrony in the face condition was correlated positively with increased positive symptoms, in line with previous research. Lee et al. (2003) demonstrated that enhanced phase synchrony in responses to targets in an oddball paradigm was correlated positively with increased positive symptoms in a sample of chronic patients with schizophrenia. Physiological and cognitive theories of schizophrenia have conceptualized the positive symptoms of schizophrenia as a compensatory mechanism, resulting from underlying disorganization that leads to an overprocessing of relevant information (Carr and Wale, 1986; Williams et al., 2003). In the present study this is supported by the significant correlations between specific items of the positive syndrome, such as delusions and auditory hallucinations, and increased phase synchrony.

For responses to stimuli in the no-face condition the increases in negative symptoms were correlated with reductions in phase synchrony, which may support our functional interpretation of the early phase synchrony responses in terms of a selection mechanism. Aspects of the negative syndrome in schizophrenia have been interpreted by several authors as homeostatic responses to compensate for the flooding of irrelevant information caused by dysfunctions in early perceptual mechanisms (Carr and Wale, 1986). Because we assume reduced phase synchrony to index such a dysfunctional perceptual mechanism, the relationship could be explained by this model.

\section{Influence of medication on EEG data}

Both the correlation with individual chlorpromazine equivalents that we computed and recent reports on related research indicate that the reductions in phase synchrony in patients with schizophrenia in our study are unlikely to reflect the effects of medication. There is evidence to suggest that dysfunctions in stimuluslocked $\gamma$-band oscillations are present in neuroleptic naive patients with schizophrenia (Gallinat et al., 2004) as well as in relatives of patients with schizophrenia (Hong et al., 2004).

\section{Relationship to previous research}

The present study demonstrates for the first time a relationship between dysfunctions in large-scale coordination of distributed neural activity and cognitive deficits in schizophrenia. Although deficits in the communication between and within functionally specialized regions of the cerebral cortex long have been regarded as critical for the understanding of the pathophysiology of schizophrenia (Friston, 1998; Andreasen, 1999; Phillips and Silverstein, 2003), we believe that the current study provides crucial evidence for this hypothesis. Disturbed communication between brain areas has been supported by previous studies that used functional magnetic resonance imaging (fMRI) (Honey et al., 2005) and anatomical measurements in patients with schizophrenia (Ardekani et al., 2003; Burns et al., 2003; Kubicki et al., 2003). However, we believe that advanced methods of EEG analysis as applied in the present study, which allow for a differentiation between local and long-range coordinating processes, provide stronger support for this hypothesis because they permit analysis of coordinating interactions over large distances in the brain with the millisecond resolution that fMRI cannot provide. Such resolution is required because synchronization of oscillatory activity in the $\beta$ - and $\gamma$-frequency bands occurs with a precision in the millisecond range (Singer, 1999).

\section{Implications for future research}

Additional studies are needed that link deficits in phase synchrony to anatomical and neurophysiological dysfunctions as well as to clinical parameters that have been associated with coordination failures in schizophrenia (Uhlhaas and Silverstein, 2005). Corticocortical connections are a likely anatomical substrate for long-range synchronization (Löwel and Singer, 1992; Roelfsema et al., 1994), and recent studies have reported evidence for reduced corticocortical connections in patients with schizophrenia (Ardekani et al., 2003; Burns et al., 2003; Kubicki et al., 2003). Moreover, there is evidence in patients with schizophrenia for abnormalities in neurotransmitter systems that have been shown to be involved in the temporal coordination of neural transmission at fast time scales, such as GABAergic neurons and NMDA receptors (Moghaddam, 2004; Lewis et al., 2005). Future research linking impaired neural synchronization to physiological and anatomical deficits would increase the utility of the concept of dysfunctional coordination in schizophrenia and potentially open up new therapeutic vistas on the disorder.

\section{References}

Andreasen NC (1999) A unitary model of schizophrenia. Arch Gen Psychiatry 52:341-351.

Ardekani BA, Nierenberg J, Hoptman MJ, Javitt DC, Lim KO (2003) MRI study of white matter diffusion anisotropy in schizophrenia. NeuroReport 14:2025-2029.

Bibbig A, Traub RD, Whittington MA (2002) Long-range synchronization of gamma and beta oscillations and the plasticity of excitatory and inhibitory synapses: a network model. J Neurophysiol 88:1634-1654.

Brovelli A, Ding M, Ledberg A, Chen Y, Nakamura R, Bressler SL (2004) Beta oscillations in a large-scale sensorimotor cortical network: directional influences revealed by Granger causality. Proc Natl Acad Sci USA 101:9849-9854.

Burns J, Job D, Bastin ME, Whalley H, Macgillivray T, Johnstone EC, Lawrie SM (2003) Structural disconnectivity in schizophrenia: a diffusion tensor imaging magnetic resonance study. Br J Psychiatry 182:439-443.

Carr V, Wale J (1986) Schizophrenia: an information processing model. Aust N Z J Psychiatry 20:136-155.

Franke GH (1995) SCL-90-R. Die Symptom-Checkliste von Derogatis, Deutsche version. Göttingen, Germany: Beltz Test.

Fries P, Neuenschwander S, Engel AK, Goebel R, Singer W (2001a) Rapid feature selective neuronal synchronization through correlated latency shifting. Nat Neurosci 4:194-200.

Fries P, Reynolds JH, Rorie AE, Desimone R (2001b) Modulation of oscillatory neuronal synchronization by selective visual attention. Science 291:1560-1563.

Friston KJ (1998) The disconnection hypothesis. Schizophr Res 30:115-125.

Gallinat J, Winterer G, Herrmann CS, Senkowski D (2004) Reduced oscillatory gamma-band responses in unmedicated schizophrenic patients indicate impaired frontal network processing. Clin Neurophysiol 115:1863-1874.

Gross J, Schmitz F, Schnitzler I, Kessler K, Shapiro K, Hommel B, Schnitzler A (2004) Modulation of long-range neural synchrony reflects temporal limitations of visual attention in humans. Proc Natl Acad Sci USA 101:13050-13055. 
Honey GD, Pomarol-Clotet E, Corlett PR, Honey RA, McKenna PJ, Bullmore ET, Fletcher PC (2005) Functional dysconnectivity in schizophrenia associated with attentional modulation of motor function. Brain 128:25972611.

Hong LE, Summerfelt A, McMahon R, Adami H, Francis G, Elliott A, Buchanan RW, Thaker GK (2004) Evoked gamma band synchronization and the liability for schizophrenia. Schizophr Res 70:293-302.

Ille N, Berg P, Scherg M (2002) Artifact correction of the ongoing EEG using spatial filters based on artifact and brain signal topographies. J Clin Neurophysiol 19:113-124.

Kay SR, Opler LA, Fiszbein A (1986) The positive and negative syndrome scale (PANSS) for schizophrenia. Schizophr Bull 13:261-276.

Kopell N, Ermentrout GB, Whittington MA, Traub RD (2000) Gamma rhythms and beta rhythms have different synchronization properties. Proc Natl Acad Sci USA 97:1867-1872.

Kubicki M, Westin CF, Nestor PG, Wible CG, Frumin M, Maier SE, Kikinis R, Jolesz FA, McCarley RW, Shenton ME (2003) Cingulate fasciculus integrity disruption in schizophrenia: a magnetic resonance diffusion tensor imaging study. Biol Psychiatry 54:1171-1180.

Lachaux JP, Rodriguez E, Martinerie J, Varela FJ (1999) Measuring phase synchrony in brain signals. Hum Brain Mapp 8:194-208.

Lee KH, Williams LM, Haig A, Gordon E (2003) Gamma (40 Hz) phase synchronicity and symptom dimensions in schizophrenia. Cognit Neuropsychiatry 8:57-71.

Lewis DA, Hashimoto T, Volk DW (2005) Cortical inhibitory neurons and schizophrenia. Nat Rev Neurosci 6:312-324.

Lindenmayer JP, Bernstein-Hyman R, Grochowski BA (1995) A new five factor model of schizophrenia. Psychiatr Q 65:299-322.

Löwel S, Singer W (1992) Selection of intrinsic horizontal connections in the visual cortex by correlated neuronal activity. Science 255:209-212.

Moghaddam B (2004) Bringing order to the glutamate chaos in schizophrenia. Neuron 40:881-884.

Mooney CM, Ferguson GA (1951) A new closure test. Can J Psychol 5:129-133.

Ohara S, Mima T, Baba K, Ikeda A, Kunieda T, Matsumoto R, Yamamoto J, Matsuhashi M, Nagamine T, Hirasawa K, Hori T, Mihara T, Hashimoto N, Salenius S, Shibasaki H (2001) Increased synchronization of cortical oscillatory activities between human supplementary motor and primary sensorimotor areas during voluntary movements. J Neurosci 21:9377-9386.

Onitsuka T, Niznikiewicz MA, Spencer KM, Frumin M, Kuroki N, Lucia LC, Shenton ME, McCarley RW (2006) Functional and structural deficits in brain regions subserving face perception in schizophrenia. Am J Psychiatry 163:455-462.

Phillips WA, Silverstein SM (2003) Convergence of biological and psychological perspectives on cognitive coordination in schizophrenia. Discussion. Behav Brain Sci 26:65-138.

Rodriguez E, Lachaux JP, Martinerie J, Renault B, Varela FJ (1999) Perception's shadow: long-distance synchronization of human brain activity. Nature 397:430-433.

Roelfsema PR, König P, Engel AK, Sireteanu R, Singer W (1994) Reduced synchronization in the visual cortex of cats with strabismic amblyopia. Eur J Neurosci 6:1645-1655.

Roelfsema PR, Engel AK, König P, Singer W (1997) Visuomotor integration is associated with zero time-lag synchronization among cortical areas. Nature 385:157-161.

Sass H, Wittchen H (2003) Diagnostisches und Statistisches Manual Psychischer Störungen, Textrevision. Bern, Switzerland: Hogrefe.

Schoffelen JM, Osstenveld R, Fries P (2005) Neuronal coherence as a mechanism of effective corticospinal interaction. Science 308:111-113.

Singer W (1999) Neuronal synchrony: a versatile code of the definition of relations? Neuron 24:49-65.

Slewa-Younan S, Gordon E, Harris AW, Haig AR, Brown KJ, Flor-Henry P, Williams LM (2004) Sex differences in functional connectivity in firstepisode and chronic schizophrenia patients. Am J Psychiatry 161:1595-1602.

Spencer KM, Nestor PG, Niznikiewicz MA, Salisbury DF, Shenton ME, McCarley RW (2003) Abnormal neural synchrony in schizophrenia. J Neurosci 23:7407-7411.

Spencer KM, Nestor PG, Perlmutter R, Niznikiewicz MA, Klump MC, Frumin M, Shenton ME, McCarley RW (2004) Neural synchrony indexes disordered perception and cognition in schizophrenia. Proc Natl Acad Sci USA 101:17288-17293

Symond MB, Harris A, Gordon E, Williams L (2005) "Gamma synchrony" in first-episode schizophrenia: a disorder of temporal connectivity? Am J Psychiatry 162:459-465.

Tallon-Baudry C, Bertrand O (1999) Oscillatory gamma activity in humans and its role in object representations. Trends Cogn Sci 3:151-162.

Tallon-Baudry C, Bertrand O, Delpuech C, Pernier J (1998) Oscillatory $\gamma$-band $(30-70 \mathrm{~Hz})$ activity induced by a short-term memory task in humans. J Neurosci 18:4244-4254.

Tallon-Baudry C, Mandon S, Freiwald WA, Kreiter AK (2004) Oscillatory synchrony in the monkey temporal lobe correlates with performance in a visual short-term memory task. Cereb Cortex 14:713-720.

Trujillo LT, Peterson MA, Kaszniak AW, Allen JJ (2005) EEG phase synchrony differences across visual perception conditions may depend on recording and analysis methods. Clin Neurophysiol 116:172-189.

Uhlhaas PJ, Silverstein SM (2005) Perceptual organization in schizophrenia spectrum disorders: a review of empirical research and associated theories. Psychol Bull 131:618-632.

Uhlhaas PJ, Silverstein SM, Phillips WA (2005) The course and clinical correlates of dysfunctions in visual perceptual organization in schizophrenia during the remission of psychotic symptoms. Schizophr Res 75:183-192.

Varela F, Lachaux JP, Rodriguez E, Martinerie J (2001) The brain web: phase synchronization and large-scale integration. Nat Rev Neurosci 2:229-239.

Williams LM, Lee K-H, Haig A, Gordon E (2003) High-frequency synchronisation in schizophrenia: too much or too little? Behav Brain Sci 26:109-110.

Woods SW (2003) Chlorpromazine equivalent doses for the newer atypical antipsychotics. J Clin Psychiatry 64:663-667. 